\title{
openheart Does pneumococcal vaccination prevent myocardial infarction and stroke in elderly adults?
}

Ole S Søgaard

To cite: Søgaard OS. Does pneumococcal vaccination prevent myocardial infarction and stroke in elderly adults?. Open Heart 2015;2:e000306 doi:10.1136/openhrt-2015000306

Accepted 3 July 2015

\section{SLinked}

- http://dx.doi.org/10 1136/openhrt-2015-000247

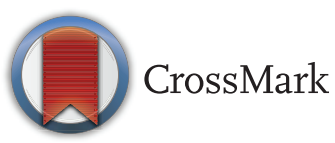

Department of Infectious Diseases, Aarhus University Hospital, Aarhus, Denmark

Correspondence to Dr Ole S Søgaard; olesoega@rm.dk
Over the past decades there has been an ongoing debate whether preventing lower respiratory tract infections through pneumococcal (polysaccharide) vaccination (PPV) could decrease the risk of venous and arterial thrombosis. This hypothesis is mainly based on findings from animal experiments indicating that systemic inflammation and infections accelerate atherosclerosis. ${ }^{1}$ Recent circumstantial evidence from an observational study in patients with persistent immune activation (eg, due to HIV infection) suggests that systemic inflammation may also accelerate atherosclerosis in humans. ${ }^{2}$ In support of this, multiple studies have shown that circulating markers of inflammation, such as $\mathrm{C}$ reactive protein, interleukin 6 and D-dimer predict the risk of arterial thromboembolic events in humans. ${ }^{3}$ Clearly, systemic inflammation is not a constant but varies in response to proinflammatory stimuli. Intermittent changes caused by acute infections have been linked to shortterm increases in the risk of vascular events in large observational studies. ${ }^{4}$ Although the pathogenesis may differ, the same associations have also been made between venous thromboembolic events, and systemic inflammation and infections. ${ }^{4} 5$ Interestingly, in addition to preventing lower respiratory tract infections, PPV-induced antibodies have been proposed to possess other potentially beneficial effects. In vitro studies have suggested molecular mimicry between the immunodominant phosphorylcholine epitopes of oxidised phospholipids of oxidised low-density lipoprotein and the phosphorylcholine moiety of Streptococcus pneumoniae. ${ }^{6}$ Studies conducted in rodents have demonstrated that immunisation with PPV induces antiphosphorylcholine binding antibodies, which some investigators believe might actively modulate atherogenesis. ${ }^{7}$

In a new article published in the Open Heart Journal, Ren et $a l^{8}$ utilised the considerable body of literature on this topic to conduct a systematic review and meta-analysis investigating the effect of PPV on risk of acute coronary syndrome (ACS) and stroke events in adults.

In this study the authors first attempted to identify randomised trials describing the effect of PPV on myocardial infarction and/ or stroke events. After reviewing 1041 unique search results, the authors found that none of these were suitable for data extraction due to uncertainty over whether the events included in the studies were ischaemic in nature. Therefore, Ren et al based their review and two-part meta-analysis on nine observational studies selected from a total of 263 unique search results. In part one of their meta-analysis, which included 230426 patients from eight observational studies, the authors found that PPV was associated with significantly lower odds of ACS in the population aged 65 years and above (pooled ratio of 0.83 (95\% CI 0.71 to 0.97 ) in favour of PPV). However, when the age restriction was not applied (ie, allowing persons younger than 65 years of age to be included), the analysis produced a pooled ratio of $0.86(95 \%$ CI 0.73 to 1.01 ) and, thus, did not quite reach statistical significance. In part two of their meta-analysis, Ren et al found that PPV was not associated with a lower risk of stroke in four observational studies consisting of a total of 192210 patients (pooled ratio of 0.96 (95\% CI 0.87 to 1.05$)$ with age restriction $>65$ years).

A $17 \%$ reduction in ACS risk among PPVvaccinated compared with PPV-unvaccinated individuals is potentially a clinically relevant and important finding. Of note, a prime-boost regimen in which a 13 valent pneumococcal conjugate vaccine (PCV) was used to 'prime' the immune system prior to 'boosting' with the 23 valent PPV is now recommended in many countries. However, if the risk reduction is real, the broad use of pneumococcal 
vaccination to prevent ACS is very likely to be cost-effective regardless of whether or not PPV is preceded by PCV.

Despite the authors' impressive efforts to enforce stringent and rigorous inclusion criteria in the selection studies for their analysis, considerable heterogeneity persists among the included studies. Follow-up time varied between the studies from 3 months to several years. Not surprisingly, baseline data also varied widely between the studies. They varied in magnitude as well as in quality for average age, gender distribution, history of ischaemic events, and other risk factors such as smoking and diabetes. Importantly, two of eight included studies reported an approximately $50 \%$ reduction in risk of ACS among PPV-vaccinated compared with PPV-unvaccinated individuals. $^{9}{ }^{10}$ This striking effect of PPV on ACS risk was far greater than what was reported in the other six studies. ${ }^{8}$ It is also reminiscent of the accounts of approximately $50 \%$ reductions in all-cause mortality of elderly adults that were previously published for influenza vaccination but later questioned due to suspicion of healthy-user bias and confounding by indication. ${ }^{11}{ }^{12}$ In other words, influenza or PPV immunisation may be a marker for factors such as diet, lifestyle and exercise that are not documented in the available data sets but that are known to be associated over the long term with risk of atherosclerosis and myocardial infarction. Missing information on known risk factors and/or uneven distribution of unknown risk for the outcome in question may lead to biased estimates. The authors rightfully point out that in some settings PPV is selectively administered to individuals with chronic conditions (ie, who are also at higher risk of ACS and stroke), which would bias the results in the opposite direction (ie, towards underestimating the protective effect of immunisation).

In summary, the interesting and well-conducted review and meta-analyses by Ren et al suggest a significant protective effect from PPV immunisation on risk of ACS. However, their work also highlights the challenges associated with deciphering observational studies reporting the impact of non-routine immunisation on outcomes not directly related to the infectious condition(s) targeted by the vaccine given. Heterogeneity in study designs, lack of randomised trials and potential bias due to a healthy-user effect, are likely to impact the overall estimate form by Ren et al. The key question remains whether pneumococcal vaccination prevents myocardial infarction and stroke in elderly adults. The ultimate test of a causal relationship between pneumococcal immunisation and ACS is a randomised clinical trial investigating the effects of PPV on ischaemic events as the primary outcome, which, according to the authors, is ongoing. Until those results become available, PPV should first and foremost be considered a tool to reduce the risk of pneumococcal disease in at-risk adults.

Competing interests None declared.

Provenance and peer review Commissioned; internally peer reviewed.

Open Access This is an Open Access article distributed in accordance with the Creative Commons Attribution Non Commercial (CC BY-NC 4.0) license, which permits others to distribute, remix, adapt, build upon this work noncommercially, and license their derivative works on different terms, provided the original work is properly cited and the use is non-commercial. See: http:// creativecommons.org/licenses/by-nc/4.0/

\section{REFERENCES}

1. Libby $P$, Ridker $P M$, Maseri A. Inflammation and atherosclerosis. Circulation 2002;105:1135-43.

2. Duprez DA, Neuhaus J, Kuller LH, et al. Inflammation, coagulation and cardiovascular disease in HIV-infected individuals. PLOS ONE 2012;7:e44454.

3. Krintus M, Kozinski M, Kubica J, et al. Critical appraisal of inflammatory markers in cardiovascular risk stratification. Crit Rev Clin Lab Sci 2014;51:263-79.

4. Smeeth L, Thomas SL, Hall AJ, et al. Risk of myocardial infarction and stroke after acute infection or vaccination. $\mathrm{N}$ Engl J Med 2004;351:2611-18.

5. Smeeth L, Cook C, Thomas S, et al. Risk of deep vein thrombosis and pulmonary embolism after acute infection in a community setting. Lancet 2006;367:1075-9.

6. Binder CJ, Horkko S, Dewan A, et al. Pneumococcal vaccination decreases atherosclerotic lesion formation: molecular mimicry between Streptococcus pneumoniae and oxidized LDL. Nat Med 2003;9:736-43.

7. Caligiuri G, Khallou-Laschet J, Vandaele M, et al. Phosphorylcholine-targeting immunization reduces atherosclerosis. J Am Coll Cardiol 2007;50:540-6.

8. Ren SN, Newby D, Li SC, et al. Effect of the adult pneumococcal polysaccharide vaccine on cardiovascular disease: a systematic review and meta-analysis. Open Heart 2015;2:e000247.

9. Lamontagne F, Garant MP, Carvalho JC, et al. Pneumococcal vaccination and risk of myocardial infarction. CMAJ 2008;179:773-7.

10. Eurich DT, Johnstone JJ, Minhas-Sandhu JK, et al. Pneumococcal vaccination and risk of acute coronary syndromes in patients with pneumonia: population-based cohort study. Heart 2012;98:1072-7.

11. Jackson LA, Jackson ML, Nelson JC, et al. Evidence of bias in estimates of influenza vaccine effectiveness in seniors. Int $J$ Epidemiol 2006;35:337-44.

12. Simonsen L, Taylor RJ, Viboud C, et al. Mortality benefits of influenza vaccination in elderly people: an ongoing controversy. Lancet Infect Dis 2007;7:658-66. 